\title{
Impacts of Hyperbolic Discounting on Inventory Replenishment Policy Under Inflation
}

\author{
Yongwu ZHOU \\ School of Business Administration, South China University of Technology, Guangzhou 510641, China \\ E-mail: zyw666@hotmail.com \\ Zhaozhan LIN \\ School of Business Administration, South China University of Technology, Guangzhou 510641, China \\ E-mail: linzhaozhanscut@qq.com
}

\begin{abstract}
Considering time inconsistency and inter-temporal preference of the decision maker who is facing inter-temporal choices, this paper employs hyperbolic discounting to reflect these characteristics in governing inventory replenishment policy under inflation. The authors take the subjective perception of the decision maker and the objective indicator from the capital market into consideration. The decision maker's subjective perception includes confidence towards future value of money and anxiety to return the money, while the objective indicator is represented by the compounded discount rate. The results suggest that over the given planning horizon, with more confidence, inventory policy of larger order quantity and smaller order frequency should be adopted; with more anxiety within a threshold, inventory policy of smaller order quantity and larger order frequency should be adopted, and with more anxiety beyond this threshold, inventory policy keeps unchanged; with a larger discount rate, inventory policy of smaller order quantity and larger order frequency should be adopted.
\end{abstract}

Keywords inventory; hyperbolic discounting; time value of money; time inconsistency; deteriorating items

\section{Introduction}

The increasing consumer price index indicates the strengthening severity of inflation these years in China. We realize that on the one hand, inventory replenishment policy is greatly influenced by the external macroeconomics; on the other hand, it's also explicitly impacted by the decision maker's subjective perception, such as his or her degree of confidence towards future value of money and anxiety to return the money. Over the planning time horizon, given the objective indicator, say, the compounded discount rate, the decision maker may prefer the conservative replenishment policy, when his or her confidence perceived towards future value of money is decreasing and anxiety to return the money is increasing. Moreover, when inflation is more severe, the decision maker may not be patient and rational any more. Instead, he

Received March 19, 2015, accepted June 29, 2015

Supported by National Natural Science Foundation of China (71131003, 71201061, 71371075), the Fundamental Research Funds for the Central Universities (2014ZZ0072), Guangdong Natural Science Foundation (2014A030310212) 
or she may become anxious and eager to return the money and gain the immediate benefit by adjusting inventory replenishment policy. That's to say, the decision maker will focus on the present, instead of the future, to acquire immediate reward. How can we reflect these characteristics in the inventory replenishment model? How do the degree of confidence and anxiety change inventory replenishment policy? How can we combine the subjective perception of the decision maker with the objective indicator from the capital market, to make better inventory decisions? The traditional inventory replenishment models don't provide with us the lens through which we can look into these issues effectively.

Trading off cost and benefit over time, people actually have bounded rationality and reveal time inconsistency. That's, people's preference for future reward may change over time and the temptation of immediate reward can be considerably irresistible ${ }^{[1-5]}$. Plenty of psychological experiments and economic literature have supported these characteristics ${ }^{[6-12]}$. When making inter-temporal decisions, people outweigh the present, compared with the future. When setting inter-temporal preference, their valuation of the outcome will be more discounted over time ${ }^{[13-16]}$. The decision maker, who is making inventory replenishment policy under inflation, also shows time inconsistency and inter-temporal preference when making inter-temporal choices. The effect of time inconsistency and inter-temporal preference will be more salient when inflation is more severe. Time inconsistency and inter-temporal preference can be represented by hyperbolic discounting ${ }^{[17]}$.

For hyperbolic discounting function, its discount rate is $\gamma /(1+\alpha t)$, and a reward at future time $t$ will be discounted by the factor $(1+\alpha t)^{-\gamma / \alpha}$, where $\alpha$ and $\gamma$ are positive ${ }^{[18-19]}$. Compared with the constant discount rate under exponential discounting, the discount rate of hyperbolic discounting is declining with regard to time $t$. This feature implies time inconsistency of the decision maker when making inter-temporal decisions. Due to analytical intractability of hyperbolic discounting function, quasi-hyperbolic discounting function is popularly employed ${ }^{[20]}$. For the discrete quasi-hyperbolic discounting function, the discount factor is given as

$$
D(t)= \begin{cases}\mathrm{e}^{-\gamma t}, & t \in\left[0, \tau_{0}\right), \\ \alpha \mathrm{e}^{-\gamma t}, & t \in\left[\tau_{0}, \infty\right),\end{cases}
$$

where time interval $\left[0, \tau_{0}\right)$ is the present with exponential discounting and time internal $\left[\tau_{0}, \infty\right)$ is the future with exponential discounting multiplying an factor $\alpha(0 \leq \alpha \leq 1)$. Besides, the length of the present $\tau_{0}$ is stochastic and exponentially distributed with hazard rate $\lambda(\lambda \geq 0)$. For the continuous quasi-hyperbolic discounting function, the discount factor is given as

$$
\bar{D}(t)=\mathrm{e}^{-\lambda t} \mathrm{e}^{-\gamma t}+\left(1-\mathrm{e}^{-\lambda t}\right) \alpha \mathrm{e}^{-\gamma t}=\alpha \mathrm{e}^{-\gamma t}+(1-\alpha) \mathrm{e}^{-(\gamma+\lambda) t},
$$

implying that $\bar{D}(t)$ is a linear combination of the exponential discount factor $\mathrm{e}^{-\gamma t}$ with weight $\alpha$ and the exponential discount factor $\mathrm{e}^{-(\gamma+\lambda) t}$ with weight $(1-\alpha)$. In our inventory models to reveal the decision maker's time inconsistency, we use continuous quasi-hyperbolic discounting function only.

Hyperbolic discounting has received some applications and provided valuable insights in behavioral operations management ${ }^{[21-23]}$. Recently, for revenue-management problem, $\mathrm{Su}^{[24]}$ considered customers' emphases on the immediate cost relative to the future benefit. For service systems, Huang et al. ${ }^{[25]}$ studied customers' bounded rationality. Plambeck and Wang ${ }^{[26]}$ 
presented the optimal pricing and scheduling for an unpleasant service generating long-term future benefits. For the newsvendor model, Chen et al. ${ }^{[27]}$ analyzed the role of mental accounting on the effect of payment schemes on inventory decisions. In this paper, we research the impacts of hyperbolic discounting on inventory replenishment policy.

Literature on the economic order quantity (EOQ) models with different demand function under inflation is abounding ${ }^{[28-31]}$. All of these models are under exponential discounting, which assumes absolute rationality and neglects time inconsistency of the decision maker. For the non-deteriorating item, Trippi and Lewin ${ }^{[32]}$ initially exercised the discounted cash flow (DCF) approach to get present value of the average inventory cost over the infinite time horizon. Moon and Yun ${ }^{[33]}$ introduced the DCF approach to consider the situation where the finite planning horizon is a random variable. Hariga ${ }^{[34]}$ considered the replenishment policy with time continuous non-stationary demand over a given finite planning horizon. For the deteriorating item $^{[35-36]}$, Bose ${ }^{[37]}$ took account of the EOQ model where demand is linear time-dependent and shortages are allowed. Chung and $\mathrm{Liu}^{[38]}$ explored a line search technique to decide the optimal interval over the finite time horizon. Chung and Lin ${ }^{[39]}$ investigated optimal inventory replenishment policy without shortage and with shortages completely backlogged where demand is stable. Moon et al. ${ }^{[40]}$ took ameliorating/deteriorating items into account where demand is time-varying. Gilding ${ }^{[41]}$ formulated optimal inventory replenishment schedule subject to timedependent demand and inflation, with no restriction on the timing of the orders or the start of the shortage periods. In this paper, however, we employ hyperbolic discounting to consider time value of money for the deteriorating item over the given planning time horizon. We research the impacts of time inconsistency and inter-temporal preference of the decision maker on inventory replenishment policy. Besides, we consider the cases with stable demand where there is no shortage and there are shortages with complete backlogging.

The rest of this paper is organized as follows. Section 2 presents the description of the models. Section 3 formulates mathematical models in the case of no shortage and shortages with complete backlogging, respectively. Section 4 provides numerical examples. Section 5 comes to the conclusions and managerial insights.

\section{Model Description}

Over a given finite planning time horizon under inflation, the decision maker is facing the problem of how to determine the optimal order frequency and order quantity in each ordering cycle, so as to maximize present value of total profit. All the costs and sales revenue are discounted by continuous quasi-hyperbolic discounting . Total cost includes fixed ordering cost, procurement cost, inventory holding cost and shortages cost (if shortages are allowed); total revenue is from selling items; total profit is the difference between total revenue and total cost.

Assumptions:

1) Time horizon is finite and lead time is zero;

2) Demand rate of single items is constant and replenishment rate of items is infinite; Notation:

$H$ Finite planning time horizon;

$T$ Length of ordering cycle, decision variable; 
$n \quad$ Order frequency, decision variable, $n=H / T$;

$Q \quad$ Order quantity, decision variable;

$T_{j}$ Total elapsed time including the $j$ th ordering cycle $(j=1,2, \cdots, n)$, denoting the occurrence of the $(j+1)$ th ordering. $T_{j}=j H / n=j T$, where $T_{j}=H$ and $T_{0}=0$;

$t_{j}$ The time at which the inventory level in the $j$ th ordering cycle drops to zero in the case of shortages $(j=1,2, \cdots, n)$;

A Fixed ordering cost per ordering;

$d$ Demand rate;

$p \quad$ Sale price per unit;

$c$ Procurement cost per unit;

$h \quad$ Inventory holding cost per unit per time;

$s \quad$ Shortage cost per unit per time;

$\theta \quad$ Deterioration rate, $0<\theta \leq 1$;

$\gamma$ Compounded discount rate, net of inflation, $0 \leq \gamma \leq 1$;

$\lambda$ Hazard rate, $\lambda \geq 0$;

$\alpha$ Confidence of the decision maker towards future value of money, $0 \leq \alpha \leq 1$.

\section{Mathematical Models}

Discount factor at any time $t$ with continuous quasi-hyperbolic discounting is given as

$$
D(t)=\mathrm{e}^{-\lambda t} \mathrm{e}^{-\gamma t}+\left(1-\mathrm{e}^{-\lambda t}\right) \alpha \mathrm{e}^{-\gamma t}=\alpha \mathrm{e}^{-\gamma t}+(1-\alpha) \mathrm{e}^{-(\gamma+\lambda) t} .
$$

Let $\beta=1-\alpha, \eta=\gamma+\lambda$, then $D(t)=\alpha \mathrm{e}^{-\gamma t}+\beta \mathrm{e}^{-\eta t}$. Thus, quasi-hyperbolic discounting could be regarded as the linear combination of exponential discounting $\mathrm{e}^{-\gamma t}$ with the discounted weight $\alpha$ and exponential discounting $\mathrm{e}^{-\eta t}$ with the discounted weight $\beta$. Here, make it clear that $\alpha$ denotes confidence of the decision maker towards future value of money. A smaller $\alpha$ means that the decision maker show less confidence towards future value of money, and the same amount of money in the future will be more depreciated than that under constant discount rate. $\lambda$ presents hazard rate, which could be understood as how soon the future perceived by the decision maker will come. A larger $\lambda$ means that the sooner the future will come and the more anxiety the decision maker will have. We use parameter $\alpha$ and $\lambda$ to reflect the decision maker's time inconsistency when facing inter-temporal choices.

\subsection{No Shortage}

In this subsection, we assume that shortages are not allowed.

Figure 1 shows the pattern of inventory level without shortage over the given planning time horizon $H$.

The instantaneous inventory level $I(t)$ during the first ordering cycle is regulated by

$$
\frac{\mathrm{d} I(t)}{\mathrm{d} t}=-\theta I(t)-d, \quad 0 \leq t \leq T .
$$

For $I(T)=0$, solution to (1) will be

$$
I(t)=\frac{d\left[\mathrm{e}^{\theta(T-t)}-1\right]}{\theta}, \quad 0 \leq t \leq T
$$




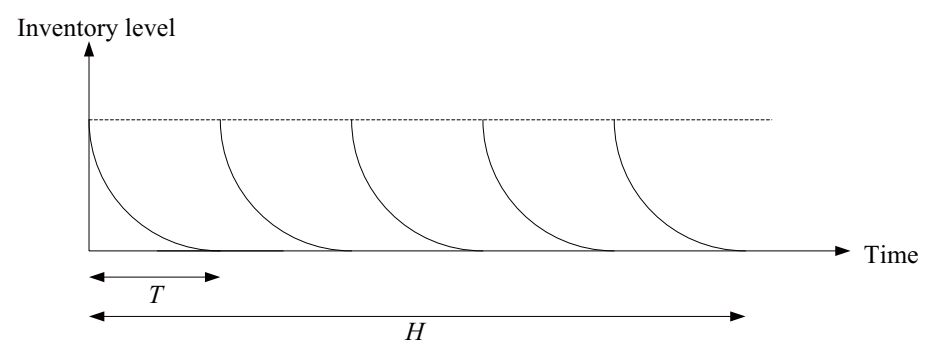

Figure 1 Inventory level of deteriorating items without shortage

Present value of holding cost during the first ordering cycle is

$$
\begin{aligned}
C_{H 1} & =h \int_{0}^{T} I(t) D(t) \mathrm{d} t \\
& =\alpha h \int_{0}^{T} \frac{d\left[\mathrm{e}^{\theta(T-t)}-1\right]}{\theta} \mathrm{e}^{-\gamma t} \mathrm{~d} t+\beta h \int_{0}^{T} \frac{d\left[\mathrm{e}^{\theta(T-t)}-1\right]}{\theta} \mathrm{e}^{-\eta t} \mathrm{~d} t \\
& =\frac{\alpha h d}{\theta}\left(\frac{\mathrm{e}^{\theta H / n}-\mathrm{e}^{-\gamma H / n}}{\theta+\gamma}+\frac{\mathrm{e}^{-\gamma H / n}-1}{\gamma}\right)+\frac{\beta h d}{\theta}\left(\frac{\mathrm{e}^{\theta H / n}-\mathrm{e}^{-\eta H / n}}{\theta+\eta}+\frac{\mathrm{e}^{-\eta H / n}-1}{\eta}\right)
\end{aligned}
$$

and present value of holding cost over the entire time horizon $H$ is

$$
\begin{aligned}
C_{H}= & \frac{\alpha h d}{\theta}\left(\frac{\mathrm{e}^{\theta H / n}-\mathrm{e}^{-\gamma H / n}}{\theta+\gamma}+\frac{\mathrm{e}^{-\gamma H / n}-1}{\gamma}\right) \sum_{j=1}^{n} \mathrm{e}^{-\gamma T_{j-1}} \\
& +\frac{\beta h d}{\theta}\left(\frac{\mathrm{e}^{\theta H / n}-\mathrm{e}^{-\eta H / n}}{\theta+\eta}+\frac{\mathrm{e}^{-\eta H / n}-1}{\eta}\right) \sum_{j=1}^{n} \mathrm{e}^{-\eta T_{j-1}} \\
= & \frac{\alpha h d}{\theta}\left(\frac{\mathrm{e}^{\theta H / n}-\mathrm{e}^{-\gamma H / n}}{\theta+\gamma}+\frac{\mathrm{e}^{-\gamma H / n}-1}{\gamma}\right) \frac{1-\mathrm{e}^{-\gamma H}}{1-\mathrm{e}^{-\gamma H / n}} \\
& +\frac{\beta h d}{\theta}\left(\frac{\mathrm{e}^{\theta H / n}-\mathrm{e}^{-\eta H / n}}{\theta+\eta}+\frac{\mathrm{e}^{-\eta H / n}-1}{\eta}\right) \frac{1-\mathrm{e}^{-\eta H}}{1-\mathrm{e}^{-\eta H / n}} .
\end{aligned}
$$

Present value of ordering cost over the entire time horizon $H$ is

$$
C_{O}=A\left(\sum_{j=1}^{n} \alpha \mathrm{e}^{-\gamma T_{j-1}}+\sum_{j=1}^{n} \beta \mathrm{e}^{-\eta T_{j-1}}\right)=\alpha A \frac{1-\mathrm{e}^{-\gamma H}}{1-\mathrm{e}^{-\gamma H / n}}+\beta A \frac{1-\mathrm{e}^{-\eta H}}{1-\mathrm{e}^{-\eta H / n}} .
$$

Present value of procurement cost over the entire time horizon $H$ is

$$
\begin{aligned}
C_{P} & =c I(0)\left(\sum_{j=1}^{n} \alpha \mathrm{e}^{-\gamma T_{j-1}}+\sum_{j=1}^{n} \beta \mathrm{e}^{-\eta T_{j-1}}\right) \\
& =c \frac{d}{\theta}\left(\mathrm{e}^{\theta H / n}-1\right)\left(\sum_{j=1}^{n} \alpha \mathrm{e}^{-\gamma T_{j-1}}+\sum_{j=1}^{n} \beta \mathrm{e}^{-\eta T_{j-1}}\right) \\
& =\frac{\alpha c d}{\theta}\left(\mathrm{e}^{\theta H / n}-1\right) \frac{1-\mathrm{e}^{-\gamma H}}{1-\mathrm{e}^{-\gamma H / n}}+\frac{\beta c d}{\theta}\left(\mathrm{e}^{\theta H / n}-1\right) \frac{1-\mathrm{e}^{-\eta H}}{1-\mathrm{e}^{-\eta H / n}} .
\end{aligned}
$$

Hence, present value of total cost over the entire time horizon $H$ is

$$
T C=C_{H}+C_{O}+C_{P} .
$$

Present value of revenue during the first ordering cycle is

$$
R=p \int_{0}^{T} d D(t) \mathrm{d} t=p d \int_{0}^{T}\left(\alpha \mathrm{e}^{-\gamma t}+\beta \mathrm{e}^{-\eta t}\right) \mathrm{d} t=\frac{\alpha p d}{\gamma}\left(1-\mathrm{e}^{-\gamma H / n}\right)+\frac{\beta p d}{\eta}\left(1-\mathrm{e}^{-\eta H / n}\right),
$$


and present value of total revenue over the entire time horizon $H$ is

$$
\begin{aligned}
T R & =\frac{\alpha p d}{\gamma}\left(1-\mathrm{e}^{-\gamma H / n}\right) \sum_{j=1}^{n} \mathrm{e}^{-\gamma T_{j-1}}+\frac{\beta p d}{\eta}\left(1-\mathrm{e}^{-\eta H / n}\right) \sum_{j=1}^{n} \mathrm{e}^{-\eta T_{j-1}} \\
& =\frac{\alpha p d}{\gamma}\left(1-\mathrm{e}^{-\gamma H / n}\right) \frac{1-\mathrm{e}^{-\gamma H}}{1-\mathrm{e}^{-\gamma H / n}}+\frac{\beta p d}{\eta}\left(1-\mathrm{e}^{-\eta H / n}\right) \frac{1-\mathrm{e}^{-\eta H}}{1-\mathrm{e}^{-\eta H / n}} .
\end{aligned}
$$

Hence, present value of total profit over the entire time horizon $H$ is

$$
T P=T R-T C
$$

Combining all the above equations and rearrange the results, we obtain

$$
\begin{aligned}
T P(n)=\alpha & \frac{p d}{\gamma}\left(1-\mathrm{e}^{-\gamma H / n}\right)-\frac{c d}{\theta}\left(\mathrm{e}^{\theta H / n}-1\right) \\
& \left.-\frac{h d}{\theta}\left(\frac{\mathrm{e}^{\theta H / n}-\mathrm{e}^{-\gamma H / n}}{\theta+\gamma}+\frac{\mathrm{e}^{-\gamma H / n}-1}{\gamma}\right)-A\right\} \frac{1-\mathrm{e}^{-\gamma H}}{1-\mathrm{e}^{-\gamma H / n}} \\
+ & \beta\left\{\frac{p d}{\eta}\left(1-\mathrm{e}^{-\eta H / n}\right)-\frac{c d}{\theta}\left(\mathrm{e}^{\theta H / n}-1\right)\right. \\
& \left.-\frac{h d}{\theta}\left(\frac{\mathrm{e}^{\theta H / n}-\mathrm{e}^{-\eta H / n}}{\theta+\eta}+\frac{\mathrm{e}^{-\eta H / n}-1}{\eta}\right)-A\right\} \frac{1-\mathrm{e}^{-\eta H}}{1-\mathrm{e}^{-\eta H / n}}
\end{aligned}
$$

Theorem 1 Present value of total profit function $T P(n)$ is concave with regard to $n$.

Before proving Theorem 1, let

$$
T C(n)=\left\{\frac{c d}{\theta}\left(\mathrm{e}^{\theta H / n}-1\right)+\frac{h d}{\theta}\left(\frac{\mathrm{e}^{\theta H / n}-\mathrm{e}^{-\gamma H / n}}{\theta+\gamma}+\frac{\mathrm{e}^{-\gamma H / n}-1}{\gamma}\right)+A\right\} \frac{1-\mathrm{e}^{-\gamma H}}{1-\mathrm{e}^{-\gamma H / n}},
$$

then we have the following lemma.

Lemma 1 Function $T C(n)$ is convex with regard to $n$.

Proof Following a similar way used by Chung and Lin ${ }^{[39]}$, one can easily derive Lemma 1. We omit the proof for brevity.

In what follows, we show the proof of Theorem 1. According to Lemma 1, $-T C(n)$ is concave with regard to $n$.

Let

$$
\begin{aligned}
& T P_{1}(n)=\left\{\frac{p d}{\gamma}\left(1-\mathrm{e}^{-\gamma H / n}\right)-\frac{c d}{\theta}\left(\mathrm{e}^{\theta H / n}-1\right)-\frac{h d}{\theta}\left(\frac{\mathrm{e}^{\theta H / n}-\mathrm{e}^{-\gamma H / n}}{\theta+\gamma}+\frac{\mathrm{e}^{-\gamma H / n}-1}{\gamma}\right)-A\right\} \frac{1-\mathrm{e}^{-\gamma H}}{1-\mathrm{e}^{-\gamma H / n}}, \\
& T P_{1}(n)=\left\{\frac{p d}{\eta}\left(1-\mathrm{e}^{-\eta H / n}\right)-\frac{c d}{\theta}\left(\mathrm{e}^{\theta H / n}-1\right)-\frac{h d}{\theta}\left(\frac{\mathrm{e}^{\theta H / n}-\mathrm{e}^{-\eta H / n}}{\theta+\eta}+\frac{\mathrm{e}^{-\eta H / n}-1}{\eta}\right)-A\right\} \frac{1-\mathrm{e}^{-\eta H}}{1-\mathrm{e}^{-\eta H / n}},
\end{aligned}
$$

then $T P_{1}(n)$ and $T P_{2}(n)$ is concave with regard to $n$, respectively.

Therefore, $T P(n)$, the linear combination of $T P_{1}(n)$ and $T P_{2}(n)$, is concave with regard to $n$. We complete the proof of Theorem 1.

Based on Theorem 1, the solution procedure to determine the optimal order frequency $n^{*}$ and present value of total profit $T P\left(n^{*}\right)$ is provided by the following algorithm.

\section{Algorithm 1}

Step 1 Input the values of all the parameters, and set $T P(0)=0$ and $n=1$;

Step 2 Calculate $T P(n)$ from (11); 
Step 3 If $T P(n)>T P(n-1)$, then set $n=n+1$ and go back to Step 2; otherwise, stop and set $n^{*}=n-1$.

Thus, we get the optimal order frequency $n^{*}$, and the optimal present value of total profit $T P\left(n^{*}\right)$. The optimal order quantity in each ordering cycle and length of ordering cycle are given as $Q^{*}=\frac{d}{\theta}\left(\mathrm{e}^{\theta H / n^{*}}-1\right), T^{*}=H / n^{*}$.

\subsection{Shortages with Complete Backlogging}

In this subsection, we assume that shortages are allowed and completely backlogged.

With shortages allowed, suppose that $(n+1)$ orderings are conducted over the given time horizon $H$. The last replenishment is made at time $t=H$, only to replenish the last shortage quantity produced in the last cycle. Assume that in each ordering cycle $\left[T_{j-1}, T_{j}\right](j=$ $1,2, \cdots, n-1)$, there are no-shortage period $K T(0<K<1)$ and shortage period $(1-K) T$. Shortages occur at time $t_{j}=(K+j-1) T(j=1,2, \cdots, n-1)$.

Figure 2 shows the pattern of inventory level with shortages completely backlogged over the given planning time horizon $H$.

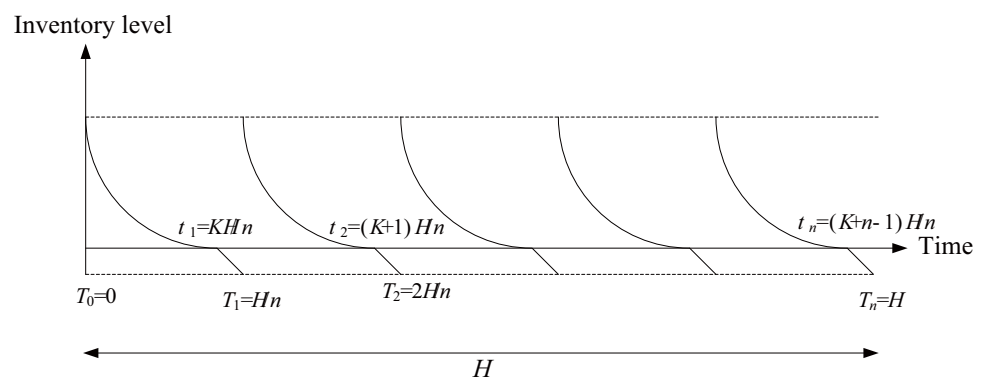

Figure 2 Inventory level of deteriorating items with shortages

The instantaneous inventory level $I(t)$ of the no-shortage period is ruled by

$$
\frac{\mathrm{d} I(t)}{\mathrm{d} t}=-\theta I(t)-d, \quad 0 \leq t \leq t_{1} .
$$

The shortages inventory level $S(t)$ is governed by

$$
\frac{\mathrm{d} S(t)}{\mathrm{d} t}=-d, \quad t_{1} \leq t \leq T .
$$

For $I(T)=0$, solutions to (12) and (13) will be

$$
\begin{aligned}
& I(t)=\frac{d\left[\mathrm{e}^{\theta\left(t_{1}-t\right)}-1\right]}{\theta}, \quad 0 \leq t \leq t_{1}, \\
& S(t)=d\left(t-t_{1}\right), \quad t_{1} \leq t \leq T .
\end{aligned}
$$

Let $I_{0}$ and $S_{0}$ be the initial inventory level and the maximum shortage quantity, respectively, then we have

$$
\begin{aligned}
I_{0} & =\frac{d}{\theta}\left(\mathrm{e}^{\theta K H / n}-1\right), \\
S_{0} & =\frac{d H(1-K)}{n} .
\end{aligned}
$$

Present value of holding cost during the first ordering cycle is

$$
C_{H 1}=h \int_{0}^{t_{1}} I(t) D(t) \mathrm{d} t
$$




$$
\begin{aligned}
= & \alpha h \int_{0}^{t_{1}} \frac{d\left[\mathrm{e}^{\theta\left(t_{1}-t\right)}-1\right]}{\theta} \mathrm{e}^{-\gamma t} \mathrm{~d} t+\beta \int_{0}^{t_{1}} \frac{d\left[\mathrm{e}^{\theta\left(t_{1}-t\right)}-1\right]}{\theta} \mathrm{e}^{-\eta t} \mathrm{~d} t \\
= & \frac{\alpha h d}{\theta}\left(\frac{\mathrm{e}^{\theta K H / n}-\mathrm{e}^{-\gamma K H / n}}{\theta+\gamma}+\frac{\mathrm{e}^{-\gamma K H / n}-1}{\gamma}\right) \\
& +\frac{\beta h d}{\theta}\left(\frac{\mathrm{e}^{\theta K H / n}-\mathrm{e}^{-\eta K H / n}}{\theta+\eta}+\frac{\mathrm{e}^{-\eta K H / n}-1}{\eta}\right),
\end{aligned}
$$

and present value of holding cost over the entire time horizon $H$ is

$$
\begin{aligned}
C_{H}= & \frac{\alpha h d}{\theta}\left(\frac{\mathrm{e}^{\theta K H / n}-\mathrm{e}^{-\gamma K H / n}}{\theta+\gamma}+\frac{\mathrm{e}^{-\gamma K H / n}-1}{\gamma}\right) \sum_{j=1}^{n} \mathrm{e}^{-\gamma T_{j-1}} \\
& +\frac{\beta h d}{\theta}\left(\frac{\mathrm{e}^{\theta K H / n}-\mathrm{e}^{-\eta K H / n}}{\theta+\eta}+\frac{\mathrm{e}^{-\eta K H / n}-1}{\eta}\right) \sum_{j=1}^{n} \mathrm{e}^{-\eta T_{j-1}} \\
= & \frac{\alpha h d}{\theta}\left(\frac{\mathrm{e}^{\theta K H / n}-\mathrm{e}^{-\gamma K H / n}}{\theta+\gamma}+\frac{\mathrm{e}^{-\gamma K H / n}-1}{\gamma}\right) \frac{1-\mathrm{e}^{-\gamma H}}{1-\mathrm{e}^{-\gamma H / n}} \\
& +\frac{\beta h d}{\theta}\left(\frac{\mathrm{e}^{\theta K H / n}-\mathrm{e}^{-\eta K H / n}}{\theta+\eta}+\frac{\mathrm{e}^{-\eta K H / n}-1}{\eta}\right) \frac{1-\mathrm{e}^{-\eta H}}{1-\mathrm{e}^{-\eta H / n}} .
\end{aligned}
$$

Present value of shortage cost during the first ordering cycle is

$$
\begin{aligned}
C_{S 1} & =s \int_{t_{1}}^{T} S(t) D(t) \mathrm{d} t \\
& =\alpha s \int_{t_{1}}^{T} d\left(t-t_{1}\right) \mathrm{e}^{-\gamma t} \mathrm{~d} t+\beta s \int_{t_{1}}^{T} d\left(t-t_{1}\right) \mathrm{e}^{-\eta t} \mathrm{~d} t \\
& =\frac{\alpha s d}{\gamma^{2}}\left[\frac{\gamma H(K-1)}{n}+\mathrm{e}^{\gamma(1-K) H / n}-1\right]+\frac{\beta s d}{\eta^{2}}\left[\frac{\eta H(K-1)}{n}+\mathrm{e}^{\eta(1-K) H / n}-1\right],
\end{aligned}
$$

and present value of shortage cost over the entire time horizon $H$ is

$$
\begin{aligned}
C_{S}= & \frac{\alpha s d}{\gamma^{2}}\left[\frac{\gamma H(K-1)}{n}+\mathrm{e}^{\gamma H(1-K) / n}-1\right] \sum_{j=1}^{n} \mathrm{e}^{-\gamma T_{j-1}} \\
& +\frac{\beta s d}{\eta^{2}}\left[\frac{\eta H(K-1)}{n}+\mathrm{e}^{\eta H(1-K) / n}-1\right] \sum_{j=1}^{n} \mathrm{e}^{-\eta T_{j-1}} \\
= & \frac{\alpha s d}{\gamma^{2}}\left[\frac{\gamma H(K-1)}{n}+\mathrm{e}^{\gamma H(1-K) / n}-1\right] \frac{1-\mathrm{e}^{-\gamma H}}{1-\mathrm{e}^{-\gamma H / n}} \\
& +\frac{\beta s d}{\eta^{2}}\left[\frac{\eta H(K-1)}{n}+\mathrm{e}^{\eta H(1-K) / n}-1\right] \frac{1-\mathrm{e}^{-\eta H}}{1-\mathrm{e}^{-\eta H / n}} .
\end{aligned}
$$

Present value of ordering cost over the entire time horizon $H$ is

$$
C_{O}=A \sum_{j=0}^{n}\left(\alpha \mathrm{e}^{-\gamma T_{j}}+\beta \mathrm{e}^{-\eta T_{j}}\right)=\alpha A \frac{\mathrm{e}^{\gamma H / n}-\mathrm{e}^{-\gamma H}}{\mathrm{e}^{\gamma H / n}-1}+\beta A \frac{\mathrm{e}^{\eta H / n}-\mathrm{e}^{-\eta H}}{\mathrm{e}^{\eta H / n}-1} .
$$

Present value of procurement cost over the entire time horizon $H$ is

$$
\begin{aligned}
C_{P} & =c I_{0} \sum_{j=1}^{n}\left(\alpha \mathrm{e}^{-\gamma T_{j-1}}+\beta \mathrm{e}^{-\eta T_{j-1}}\right)+c S_{0} \sum_{j=1}^{n}\left(\alpha \mathrm{e}^{-\gamma T_{j}}+\beta \mathrm{e}^{-\eta T_{j}}\right) \\
& =\frac{c d}{\theta}\left(\mathrm{e}^{\theta H / n}-1\right) \sum_{j=1}^{n}\left(\alpha \mathrm{e}^{-\gamma T_{j-1}}+\beta \mathrm{e}^{-\eta T_{j-1}}\right)+\frac{c d H(1-K)}{n} \sum_{j=1}^{n}\left(\alpha \mathrm{e}^{-\gamma T_{j}}+\beta \mathrm{e}^{-\eta T_{j}}\right)
\end{aligned}
$$




$$
\begin{aligned}
= & \frac{\alpha c d}{\theta}\left(\mathrm{e}^{\theta K H / n}-1\right) \frac{1-\mathrm{e}^{-\gamma H}}{1-\mathrm{e}^{-\gamma H / n}}+\frac{\beta c d}{\theta}\left(\mathrm{e}^{\theta K H / n}-1\right) \frac{1-\mathrm{e}^{-\eta H}}{1-\mathrm{e}^{-\eta H / n}} \\
& +\frac{\alpha c d H(1-K)}{n} \frac{1-\mathrm{e}^{-\gamma H}}{\mathrm{e}^{\gamma H / n}-1}+\frac{\beta c d H(1-K)}{n} \frac{1-\mathrm{e}^{-\eta H}}{\mathrm{e}^{\eta H / n}-1} .
\end{aligned}
$$

Hence, present value of total cost over the entire time horizon $H$ is given as

$$
T C=C_{H}+C_{O}+C_{P}+C_{S}
$$

Present value of revenue during the first ordering cycle is

$$
\begin{aligned}
R=p d \int_{0}^{t_{1}} D(t) \mathrm{d} t & =\alpha p d \int_{0}^{t_{1}} \mathrm{e}^{-\gamma t} \mathrm{~d} t+\beta p d \int_{0}^{t_{1}} \mathrm{e}^{-\eta t} \mathrm{~d} t \\
& =\frac{\alpha p d}{\gamma}\left(1-\mathrm{e}^{-\gamma K H / n}\right)+\frac{\beta p d}{\eta}\left(1-\mathrm{e}^{-\eta K H / n}\right),
\end{aligned}
$$

and present value total revenue over the entire time horizon $H$ is given as

$$
\begin{aligned}
T R= & \frac{\alpha p d}{\gamma}\left(1-\mathrm{e}^{-\gamma K H / n}\right) \sum_{j=1}^{n} \mathrm{e}^{-\gamma T_{j-1}}+\frac{\beta p d}{\eta}\left(1-\mathrm{e}^{-\eta K H / n}\right) \sum_{j=1}^{n} \mathrm{e}^{-\eta T_{j-1}} \\
& +\frac{p d H(1-K)}{n}\left(\sum_{j=1}^{n} \alpha \mathrm{e}^{-\gamma T_{j}}+\sum_{j=1}^{n} \beta \mathrm{e}^{-\eta T_{j}}\right) \\
= & \frac{\alpha p d}{\gamma}\left(1-\mathrm{e}^{-\gamma K H / n}\right) \frac{1-\mathrm{e}^{-\gamma H}}{1-\mathrm{e}^{-\gamma H / n}}+\frac{\beta p d}{\eta}\left(1-\mathrm{e}^{-\eta K H / n}\right) \frac{1-\mathrm{e}^{-\eta H}}{1-\mathrm{e}^{-\eta H / n}} \\
& +\frac{\alpha p d H(1-K)}{n} \frac{1-\mathrm{e}^{-\gamma H}}{\mathrm{e}^{\gamma H / n}-1}+\frac{\beta p d H(1-K)}{n} \frac{1-\mathrm{e}^{-\eta H}}{\mathrm{e}^{\eta H / n}-1} .
\end{aligned}
$$

Hence, present value of total profit over the entire time horizon $H$ is

$$
T P=T R-T C
$$

Combining all the above equations and rearrange the results, we obtain

$$
\begin{aligned}
& T P(n, K) \\
& =\alpha d\left\{\frac{p}{\gamma}\left(1-\mathrm{e}^{-\gamma K H / n}\right)-\frac{c}{\theta}\left(\mathrm{e}^{\theta K H / n}-1\right)-\frac{h}{\theta}\left(\frac{\mathrm{e}^{\theta K H / n}-\mathrm{e}^{-\gamma K H / n}}{\theta+\gamma}+\frac{\mathrm{e}^{-\gamma K H / n}-1}{\gamma}\right)\right. \\
& \left.-\frac{s}{\gamma^{2}}\left[\frac{\gamma H(K-1)}{n}+\mathrm{e}^{\gamma H(1-K) / n}-1\right]\right\} \frac{1-\mathrm{e}^{-\gamma H}}{1-\mathrm{e}^{-\gamma H / n}} \\
& +\beta d\left\{\frac{p}{\eta}\left(1-\mathrm{e}^{-\eta K H / n}\right)-\frac{c}{\theta}\left(\mathrm{e}^{\theta K H / n}-1\right)-\frac{h}{\theta}\left(\frac{\mathrm{e}^{\theta K H / n}-\mathrm{e}^{-\eta K H / n}}{\theta+\eta}+\frac{\mathrm{e}^{-\eta K H / n}-1}{\eta}\right)\right. \\
& \left.-\frac{s}{\eta^{2}}\left[\frac{\eta H(K-1)}{n}+\mathrm{e}^{\eta H(1-K) / n}-1\right]\right\} \frac{1-\mathrm{e}^{-\eta H}}{1-\mathrm{e}^{-\eta H / n}}+\alpha(p-c) \frac{d H(1-K)}{n} \frac{1-\mathrm{e}^{-\gamma H}}{\mathrm{e}^{\gamma H / n}-1} \\
& +\beta(p-c) \frac{d H(1-K)}{n} \frac{1-\mathrm{e}^{-\eta H}}{\mathrm{e}^{\eta H / n}-1}-\alpha A \frac{\mathrm{e}^{\gamma H / n}-\mathrm{e}^{-\gamma H}}{\mathrm{e}^{\gamma H / n}-1}-\beta A \frac{\mathrm{e}^{\eta H / n}-\mathrm{e}^{-\eta H}}{\mathrm{e}^{\eta H / n}-1} .
\end{aligned}
$$

Theorem 2 For the given $n$, present value of total profit function $\operatorname{TP}(n, K)$ is concave with regard to $K$.

Proof Let

$$
u_{\gamma}=\frac{1-\mathrm{e}^{-\gamma H}}{1-\mathrm{e}^{-\gamma H / n}}, \quad u_{\eta}=\frac{1-\mathrm{e}^{-\eta H}}{1-\mathrm{e}^{-\eta H / n}}, \quad v_{\gamma}=\frac{1-\mathrm{e}^{-\gamma H}}{\mathrm{e}^{\gamma H / n}-1}, \quad v_{\eta}=\frac{1-\mathrm{e}^{-\eta H}}{\mathrm{e}^{\eta H / n}-1} .
$$


For the given $n$, we have

$$
\begin{aligned}
& \frac{\partial T P}{\partial K} \\
= & \frac{u_{\gamma} \alpha d H}{n}\left\{p \mathrm{e}^{-\gamma K H / n}-c \mathrm{e}^{\theta K H / n}-h\left(\frac{\mathrm{e}^{\theta K H / n}-\mathrm{e}^{-\gamma K H / n}}{\theta+\gamma}\right)-\frac{s}{\gamma}\left[1-\mathrm{e}^{\gamma H(1-K) / n}\right]\right\} \\
& +\frac{u_{\eta} \beta d H}{n}\left\{p \mathrm{e}^{-\eta K H / n}-c \mathrm{e}^{\theta K H / n}-h\left(\frac{\mathrm{e}^{\theta K H / n}-\mathrm{e}^{-\eta K H / n}}{\theta+\eta}\right)-\frac{s}{\eta}\left[1-\mathrm{e}^{\eta H(1-K) / n}\right]\right\} \\
& -\frac{v_{\gamma} \alpha d(p-c) H}{n}-\frac{v_{\eta} \beta d(p-c) H}{n}, \\
& \frac{\partial^{2} T P}{\partial K^{2}} \\
= & -\frac{u_{\gamma} \alpha d H^{2}}{n^{2}}\left\{\frac{p}{\gamma} \mathrm{e}^{-\gamma K H / n}+c \theta \mathrm{e}^{\theta K H / n}+h\left(\frac{\theta \mathrm{e}^{\theta K H / n}+\gamma \mathrm{e}^{-\gamma K H / n}}{\theta+\gamma}\right)+s \mathrm{e}^{\gamma H(1-K) / n}\right\} \\
& -\frac{u_{\eta} \beta d H^{2}}{n^{2}}\left\{\frac{p}{\eta} \mathrm{e}^{-\eta K H / n}+c \theta \mathrm{e}^{\theta K H / n}+h\left(\frac{\theta \mathrm{e}^{\theta K H / n}+\eta \mathrm{e}^{-\eta K H / n}}{\theta+\eta}\right)+s \mathrm{e}^{\eta H(1-K) / n}\right\} .
\end{aligned}
$$

Therefore, for the given $n, \frac{\partial^{2} T P}{\partial K^{2}}<0, T P(n, K)$ is concave with regard to $K$. We complete the proof of Theorem 2 .

Based on Theorem 2, the solution procedure to determine the optimal order frequency $n^{*}$ and its corresponding $K^{*}$ is provided by the following algorithm.

\section{Algorithm 2}

Step 1 Input the values of all the parameters, and set $T P(0)=0$ and $n=1$;

Step 2 Find the solution to (29). Let $K=K(n)$ denote the solution;

Step 3 Calculate $T P(n, K)$ from $(28)$. Let $T P(n, K(n))$ denote the result;

Step 4 If $T P(n, K(n))>T P(n-1, K(n-1))$, then set $n=n+1$ and go back to Step 2; otherwise, stop and set $n^{*}=n-1, K^{*}=K(n-1)$.

Thus, we get the optimal order frequency $n^{*}$, its corresponding $K^{*}$ and the optimal present value of total profit $T P\left(n^{*}, K^{*}\right)$. The optimal order quantity in each ordering cycle, backorder quantity and length of ordering cycle are given as $Q^{*}=\frac{d}{\theta}\left(\mathrm{e}^{\theta K^{*} H / n^{*}}-1\right), B Q^{*}=$ $\frac{d\left(1-K^{*}\right) H}{n^{*}}, T^{*}=H / n^{*}$.

\section{Numerical Example}

For the hazard rate, $\lambda \geq 0$, assume $\lambda \in\{0,1,12,52,365, \infty\}$. That's, if $\lambda=0$, the future never comes; if $\lambda=1,12,52,365$, the future arrives on average once a year, once a month, once a week, once a day, respectively; if $\lambda=\infty$, the future arrives instantaneously. Values of other parameters are given as: $H=1$ year, $A=50 \$$ /ordering, $d=8000$ units/year, $p=1 \$ /$ unit, $c=0.4$ $\$ /$ unit, $h=0.15 \$ /$ unit/time, $\theta=0.02, \gamma=0.02, \alpha=0.7, \lambda=12$.

\subsection{Example 1: No Shortage}

Following the solution procedure provided in subsection 3.1, we obtain the following optimal solutions in this case: $n^{*}=6, T^{*}=2$ month, $Q^{*}=1336$ units, $T P^{*}=3035 \$$.

To observe the impact of parameter $\alpha$ on the optimal results, we change its value from 0 to 1 in step by 0.2 . The results are showed in Table 1 . 
Table 1 Impact of parameter $\alpha$ on the optimal results in the case of no shortage

\begin{tabular}{ccccc}
\hline$\alpha$ & $n^{*}$ & $T^{*}($ month $)$ & $Q^{*}$ & $T P^{*}$ \\
\hline 1 & 4 & 3 & 2005 & 4389 \\
0.8 & 5 & 2.4 & 1603 & 3476 \\
0.6 & 7 & 1.714 & 1145 & 2602 \\
0.4 & 9 & 1.333 & 890 & 1758 \\
0.2 & 12 & 1 & 667 & 948 \\
0 & 22 & 0.545 & 364 & 199 \\
\hline
\end{tabular}

Parameter $\alpha$ mirrors the confidence of the decision maker towards future value of money. Obviously, Table 1 displays that with the increase of parameter $\alpha$, the optimal order frequency decreases, order quantity in each ordering cycle increases, and present value of total profit increases. If the decision maker reveals more confidence towards future value of money, inventory replenishment policy of larger order quantity in each ordering cycle and smaller order frequency should be employed to govern inventory.

Worthy of noting is that when $\alpha=1$, the present model coincides with the counterpart under exponential discounting. Table 1 shows that the optimal present value of total profit under exponential discounting is greater than that under quasi-hyperbolic discounting. The main reason stems from the assumption of exponential discounting that the decision maker is absolutely rational. Under quasi-hyperbolic discounting, the decreasing confidence towards future value of money leads to conservative ordering.

We also observe the impact of parameter $\lambda$ on the optimal results, which is displayed in Table 2.

Table 2 Impact of parameter $\lambda$ on the optimal results in the case of no shortage

\begin{tabular}{ccccc}
\hline$\lambda$ & $n^{*}$ & $T^{*}($ month $)$ & $Q^{*}$ & $T P^{*}$ \\
\hline 0 & 4 & 3 & 2005 & 4389 \\
1 & 5 & 2.4 & 1603 & 3823 \\
12 & 6 & 2 & 1336 & 3035 \\
52 & 6 & 2 & 1336 & 2911 \\
365 & 6 & 2 & 1336 & 2873 \\
$\infty$ & 6 & 2 & 1336 & 2866 \\
\hline
\end{tabular}

Parameter $\lambda$, the hazard rate with exponential distribution, reflects how soon the future perceived by the decision maker will come. If $\lambda$ doesn't exceed a threshold, with the increase of $\lambda$, the optimal order frequency increases, the order quantity in each ordering cycle decreases, and present value of total profit decreases; and that if $\lambda$ is greater than this threshold, with the increase of $\lambda$, both the optimal order frequency and order quantity in each ordering cycle remain unchanged, but present value of total profit keeps decreasing further, though the decreasing pace slows down. Known from Table 2 , the threshold in this case is $\lambda=12$, indicating the arrival of the future on average once a month is the decision maker's upper limit. The decision maker will become indifferent to the hazard rate if $\lambda$ is greater than this threshold and $\lambda$ will exert very little influence on inventory replenishment policy in this context. This kind of perception, a presentation of anxiety to return the money, actually prevails among the decision makers, 
especially when inflation is quite severe. Therefore, if the decision maker reveals more anxiety within a certain limit to return the money, inventory replenishment policy of smaller order quantity in each ordering cycle and larger order frequency should be employed, in order to lower its influence and generate more profit; otherwise, if this anxiety to return the money is beyond this upper limit, the decision maker become indifferent to its influence and inventory replenishment policy will not be changed any more.

Specially, when $\lambda=0$, the present model degenerates into its counterpart under exponential discounting. The optimal present value of total profit under exponential discounting is greater than that under quasi-hyperbolic discounting. This is rooted in the ground that under quasihyperbolic discounting, the anxiety to return the money induces the decision maker to acquire immediate reward and gain instantaneous satisfaction facing.

Likewise, the impact of parameter $\gamma$ on the optimal results is also observed and shown in Table 3.

Table 3 Impact of parameter $\gamma$ on the optimal results in the case of no shortage

\begin{tabular}{ccccc}
\hline$\gamma$ & $n^{*}$ & $T^{*}($ month $)$ & $Q^{*}$ & $T P^{*}$ \\
\hline 0.001 & 6 & 2 & 1336 & 3068 \\
0.01 & 6 & 2 & 1336 & 3053 \\
0.05 & 6 & 2 & 1336 & 2984 \\
0.1 & 6 & 2 & 1336 & 2901 \\
0.5 & 7 & 1.714 & 1145 & 2337 \\
1 & 9 & 1.333 & 890 & 1825 \\
\hline
\end{tabular}

Table 3 shows that for $\gamma \in(0,0.1)$, the optimal order frequency and order quantity in each ordering cycle remain unchanged, which means that time value of money has little influence on inventory replenishment policy in this situation; for $\gamma \in[0.1,1]$, with the increase of $\gamma$, the optimal order frequency increases and order quantity in each ordering cycle decreases. That's to say, when inflation is more severe, inventory replenishment policy of smaller order quantity in each ordering cycle and larger order frequency should be adopted.

\subsection{Example 2: Shortages with Complete Backlogging}

Suppose that $s=1.2 \$ /$ unit/time and that all the other parameters keep the same as in the Example 1. Following the solution procedure provided in subsection 3.2, we have the following optimal results:

$n^{*}=5, K^{*}=0.7925, T^{*}=2.4$ month, $Q^{*}=1270$ units, $B Q^{*}=332$ units, $T P^{*}=3034 \$$.

The impact of parameter $\alpha$ is shown in Table 4 .

From Table 4, similarly, it's obvious that with the increase of parameter $\alpha$, the optimal order frequency decreases, order quantity in each ordering cycle increases and present value of total profit increases. In other words, if the decision maker reveals more confidence towards future value of money, inventory replenishment policy of larger order quantity in each ordering cycle and smaller order frequency should be employed.

Fraction of shortages plays the role on adjusting inventory level. With the increase of $\alpha$, $K^{*}$ increases. That's to say, the more confidence towards future value of money, the smaller fraction of shortages in each ordering cycle will be. This provides the decision maker with the 
leverage on which inventory can be adjusted to be consumed within the nearer future, when confidence towards future value of money strengthens.

Table 4 Impact of parameter $\alpha$ on the optimal results in the case of shortages

\begin{tabular}{ccccccc}
\hline$\alpha$ & $n^{*}$ & $K^{*}$ & $T^{*}($ month $)$ & $Q^{*}$ & $B Q^{*}$ & $T P^{*}$ \\
\hline 1 & 3 & 0.8796 & 4 & 2352 & 321 & 4361 \\
0.8 & 5 & 0.8241 & 2.4 & 1321 & 281 & 3468 \\
0.6 & 6 & 0.7601 & 2 & 1015 & 320 & 2613 \\
0.4 & 8 & 0.6939 & 1.5 & 695 & 306 & 1787 \\
0.2 & 10 & 0.6270 & 1.2 & 502 & 298 & 990 \\
0 & 17 & 0.5761 & 0.7059 & 271 & 199 & 235 \\
\hline
\end{tabular}

The impact of parameter $\lambda$ is shown in Table 5 .

Table 5 Impact of parameter $\lambda$ on the optimal results in the case of shortages

\begin{tabular}{ccccccc}
\hline$\lambda$ & $n^{*}$ & $K^{*}$ & $T^{*}($ month $)$ & $Q^{*}$ & $B Q^{*}$ & $T P^{*}$ \\
\hline 0 & 3 & 0.8796 & 4 & 2352 & 321 & 4361 \\
1 & 4 & 0.8433 & 3 & 1690 & 313 & 3816 \\
12 & 5 & 0.7925 & 2.4 & 1270 & 332 & 3034 \\
52 & 6 & 0.7905 & 2 & 1055 & 279 & 2912 \\
365 & 6 & 0.9167 & 2 & 1224 & 111 & 2857 \\
$\infty$ & 6 & 0.9429 & 2 & 1259 & 76 & 2849 \\
\hline
\end{tabular}

From Table 5, similarly, we know that generally, if $\lambda$ doesn't exceed a threshold, with the increase of $\lambda$, the optimal order frequency increases, the order quantity in each ordering cycle decreases, and present value of total profit decreases; and that if $\lambda$ is greater than this threshold, with the increase of $\lambda$, both the optimal order frequency and order quantity in each ordering cycle remain unchanged, but present value of total profit keeps decreasing further with a slower pace. Known from Table 5, the threshold in this case is $\lambda=52$, indicating the arrival of the future on average once a week is the decision maker's upper limit. In other words, if the decision maker reveals more anxiety within a certain threshold to return the money, inventory replenishment policy of smaller order quantity in each ordering cycle and larger order frequency should be employed to lower its influence and generate more profit; otherwise, if this anxiety to return the money is beyond this upper threshold, the decision maker become indifferent to its influence and inventory replenishment policy will not be changed any more.

If $\lambda$ doesn't exceed this threshold, with the increase of parameter $\lambda, K^{*}$ decreases; if $\lambda$ is greater than this threshold, with the increase of parameter $\lambda, K^{*}$ increases. That's to say, if the decision maker reveals more anxiety within this threshold to return the money, fraction of shortages increases; otherwise, if this anxiety to return the money is beyond this upper threshold, fraction of shortages decreases. The leverage of fraction of shortages on inventory level can be adjusted based on the decision maker's anxiety to return the money.

The impact of parameter $\gamma$ is shown in Table 6 . 
Table 6 Impact of parameter $\gamma$ on the optimal results in the case of shortages

\begin{tabular}{ccccccc}
\hline$\gamma$ & $n^{*}$ & $K^{*}$ & $T^{*}($ month $)$ & $Q^{*}$ & $B Q^{*}$ & $T P^{*}$ \\
\hline 0.001 & 5 & 0.7950 & 2.4 & 1274 & 328 & 3067 \\
0.01 & 5 & 0.7938 & 2.4 & 1272 & 330 & 3051 \\
0.05 & 6 & 0.7897 & 2 & 1054 & 280 & 2986 \\
0.1 & 6 & 0.7835 & 2 & 1046 & 289 & 2906 \\
0.5 & 7 & 0.7451 & 1.7143 & 853 & 291 & 2362 \\
1 & 8 & 0.7127 & 1.5 & 713 & 287 & 1865 \\
\hline
\end{tabular}

Table 6 shows that on the whole, with the increase of the compounded discount rate $\gamma$, the optimal order frequency increases, order quantity in each ordering cycle decreases, and present value of total profit decreases. This result is mainly originated from the depreciation of money as time goes on. In other words, when the effect of inflation strengthens, inventory replenishment policy of smaller order quantity in each ordering cycle and larger order frequency should be adopted.

With the increase of $\gamma, K^{*}$ decreases. That's to say, with greater depreciation of money in the future, the fraction of shortages in each ordering cycle will be larger. The decision maker can adjust the level of shortages according the degree of inflation so as to maximize profit.

\section{Conclusions}

Trading off cost and benefit over time, people reveal time inconsistency and are not patient enough towards their investment in the future. They have the inclination to gain immediate reward, which may produce high instantaneous fulfillment but low long-term benefit. Thus, we employ hyperbolic discounting to reflect this time inconsistency and inter-temporal preference in inventory replenishment policy. Under hyperbolic discounting, we combine the subjective perception of the decision maker with the objective indicator from the capital market to research inventory replenishment policy. From our research, we come to the conclusions as follows.

For the impact of confidence towards future value of money, if embracing more confidence, the decision maker should employ the inventory replenishment policy of larger order quantity in each ordering cycle and smaller order frequency, which can generate more profit. If shortages are allowed, fraction of shortages in each ordering circle should decrease with the increase of this confidence.

For the impact of anxiety to return the money, if anxiety within a certain threshold, the decision maker should employ the inventory replenishment policy of smaller order quantity in each ordering cycle and larger order frequency; if anxiety beyond this threshold, the decision maker will become indifferent to its impact and the inventory replenishment policy will keep unchanged. If shortages are allowed, fraction of shortages in each ordering circle should decrease with the increase of the anxiety within a certain threshold; fraction of shortages should increase with the increase of the anxiety beyond this threshold. The threshold of the anxiety in the case of shortages can be different from that in the case of no shortage.

For the impact of the compounded discount rate, if inflation becomes more severe, the 
decision maker should employ the inventory replenishment policy of smaller order quantity in each ordering cycle and larger order frequency, which can generate more profit. If shortages are allowed, fraction of shortages in each ordering circle should increase with the intensifying of inflation.

In all the cases, the optimal present value of total profit under hyperbolic discounting is smaller than that under exponential discounting. This result originates from the assumption of absolute rationality and the neglect of time inconsistency under exponential discounting, when the decision maker is facing time tradeoff to make inter-temporal decisions.

Hyperbolic discounting provides us with the insights into which we can shed light on the time inconsistency and inter-temporal preference of the decision maker facing inter-temporal choices to schedule inventory replenishment policy. Taking these insights into accounts, hyperbolic discounting impacts inventory replenishment policy.

\section{References}

[1] Adams A, Cherchye L, Rock B D, et al. Consume now or later? Time inconsistency, collective choice, and revealed preference. American Economic Review, 2014, 104(12): 4147-4183.

[2] Baucells M, Heukamp F H. Probability and time trade-off. Management Science, 2012, 58(4): 831-842.

[3] Ebert S, Strack P. Until the bitter end: On prospect theory in a dynamic context. American Economic Review, 2015, 105(4): 1618-1633.

[4] Jackson M O, Yariv L. Present bias and collective dynamic choice in the lab. American Economic Review, 2014, 104(12): 4184-4204.

[5] Read D, Frederick S, Orsel B, et al. Four score and seven years from now: The data/delay effect in temporal discounting. Management Science, 2005, 51(9): 1326-1335.

[6] Ainslie G. Pure hyperbolic discount curves predict "eyes open" self-control. Theory and Decision, 2012, 73(1): 3-34.

[7] Biais B, Hombert J, Weill P O. Equilibrium pricing and trading volume under preference uncertainty. Review of Economic Studies, 2014, 81: 1401-1437.

[8] Frederick S, Loewenstein G, O'Donoghue T. Time discounting and time preference: A critical review. Journal of Economic Literature, 2002, 40(2): 351-401.

[9] Huang Y S, Hsu C Z. An anticipative hyperbolic discount utility on intertemporal decision making. European Journal of Operational Research, 2008, 184(1): 281-290.

[10] Olea J L M, Strzalecki T. Axiomatization and measurement of quasi-hyperbolic discounting. Quarterly Journal of Economics, 2014, 129(3): 1449-1499.

[11] Toubia O, Johnson E, Evgeniou T, et al. Dynamic experiments for estimating preferences: An adaptive method of eliciting time and risk parameters. Management Science, 2013, 59(3): 613-640.

[12] Scholten M, Read D. Discounting by intervals: A generalized model of intertemporal choice. Management Science, 2006, 52(9): 1424-1436.

[13] Abdellaoui M, Diecidue E, Öncüler A. Risk preferences at different time periods: An experimental investigation. Management Science, 2011, 57(5): 975-987.

[14] Attema A E, Bleichrodt H, Rhode K I M, et al. Time-tradeoff sequences for analyzing discounting and time inconsistency. Management Science, 2010, 56(11): 2015-2030.

[15] Sayman S, Öncüler A. An investigation of time-inconsistency. Management Science, 2009, 55(3): 470-482.

[16] Zauberman G, Kim B K, Malkoc S A, et al. Discounting time and time discounting: Subjective time perception and intertemporal preferences. Journal of Marketing Research, 2009, 46(4): 543-556.

[17] Laibson D. Golden eggs and hyperbolic discounting. Quarterly Journal of Economics, 1997, 112(2): 443477.

[18] Angeletos G M, Laibson D, Repetto A, et al. The hyperbolic consumption model: Calibration, simulation, and empirical evaluation. Journal of Economic Perspectives, 2001, 15(3): 47-68.

[19] Rohde K I M. The hyperbolic factor: A measure of time inconsistency. Journal of Risk and Uncertainty, 2010, 41(2): 125-140. 
[20] Harris C, Laibson D. Instantaneous gratification. Quarterly Journal of Economics, 2013, 128(1): 205-248.

[21] Bendoly E, Croson R, Goncalves P, et al. Bodies of knowledge for research in behavioral operations. Production and Operations Management, 2010, 19(4): 434-452.

[22] Gans N, Croson R. Introduction to the special issue on behavioral operations. Manufacturing \& Service Operations Management, 2008, 10(4): 563-565.

[23] Gino F, Pisano G. Toward a theory of behavioral operations. Manufacturing \& Service Operations Management, 2008, 10(4): 676-691.

[24] Su X. Bounded rationality in newsvendor models. Manufacturing \& Service Operations Management, 2008, 10(4): $566-589$.

[25] Huang T, Allon G, Bassamboo A. Bounded rationality in service systems. Manufacturing \& Service Operations Management, 2013, 15(2): 263-279.

[26] Plambeck E L, Wang Q. Implications of hyperbolic discounting for optimal pricing and scheduling of unpleasant services that generate future benefits. Management Science, 2013, 59(8): 1927-1946.

[27] Chen L, Kök A G, Tong J D. The effect of payment schemes on inventory decisions: The role of mental accounting. Management Science, 2013, 59(2): 436-451.

[28] Hariga M A. Optimal EOQ models for deteriorating items with time-varying demand. Journal of the Operational Research Society, 1996, 47(10): 1228-1246.

[29] Benkherouf L, Mahmoud M G. On an inventory model for deteriorating items with increasing time-varying demand and shortages. Journal of the Operational Research Society, 1996, 47(1): 188-200.

[30] Yang H L, Teng J T, Chern M S. Deterministic inventory lot-size models under inflation with shortages and deterioration for fluctuating demand. Naval Research Logistics, 2001, 48(2): 144-158.

[31] Yang H L, Teng J T, Chern M S. An inventory model under inflation for deteriorating items with stockdependent consumption rate and partial backlogging shortages. International Journal of Production Economics, 2010, 123(1): 8-19.

[32] Trippi R R, Lewin D E. A present value formulation of the classical EOQ problem. Decision Science, 1974, 5(1): 30-35.

[33] Moon I, Yun W. An economic order quantity model with a random planning horizon. The Engineering Economist, 1993, 39(1): 77-86.

[34] Hariga M A. Economic analysis of dynamic inventory models with non-stationary costs and demand. International Journal of Production Economics, 1994, 36(3): 255-266.

[35] Bakker M, Riezebos J, Teunter R H. Review of inventory systems with deterioration since 2001. European Journal of Operational Research, 2012, 221(2): 275-284.

[36] Pentico D W, Drake M J. A survey of deterministic models for the EOQ and EPQ with partial backordering. European Journal of Operational Research, 2011, 214(2): 179-198.

[37] Bose S, Goswami A, Chaudhuri A, et al. An EOQ model for deteriorating items with linear time-dependent demand rate and shortages under inflation and time discounting. Journal of the Operational Research Society, 1995, 46(6): 771-782.

[38] Chung K J, Liu J, Tsai S F. Inventory systems for deteriorating items taking account of time value. Engineering Optimization, 1997, 27(4): 303-320.

[39] Chung K J, Lin C N. Optimal inventory replenishment models for deteriorating items taking account of time discounting. Computers \& Operations Research, 2001, 28(1): 67-83.

[40] Moon I, Giri B C, Ko B. Economic order quantity models for ameliorating/deteriorating items under inflation and time discounting. European Journal of Operational Research, 2005, 162(3): 773-785.

[41] Gilding B H. Inflation and the optimal inventory replenishment schedule within a finite planning horizon. European Journal of Operational Research, 2014, 234(3): 683-693. 